\title{
Skp2 is associated with paclitaxel resistance in prostate cancer cells
}

\author{
YEGUO YANG $^{1,2}$, YI LU ${ }^{1,2}$, LIHUI WANG ${ }^{1,2}$, ATSUSHI MIZOKAMI ${ }^{3}$, \\ EVAN T. KELLER ${ }^{4}$, JIAN ZHANG ${ }^{1,2,4}$ and JIEJUN FU ${ }^{1,2}$ \\ ${ }^{1}$ Key Laboratory of Longevity and Aging-Related Diseases, Ministry of Education; \\ ${ }^{2}$ Center for Translational Medicine, Guangxi Medical University, Nanning, Guangxi 530021, P.R. China; \\ ${ }^{3}$ Department of Urology, Kanazawa University, Kanazawa 920-8641, Japan; ${ }^{4}$ Department of Urology \\ and Pathology, School of Medicine, University of Michigan, Ann Arbor, MI 48109, USA
}

Received January 19, 2016; Accepted February 24, 2016

DOI: 10.3892/or.2016.4809

\begin{abstract}
Prostate cancer is the most commonly diagnosed tumor in men in the United States. Patients with hormonerefractory prostate cancer are often treated with paclitaxel, but most of them eventually develop drug resistance. S-phase kinase associated protein $2(\mathrm{Skp} 2)$ is a component of the SCF (Skp1-Cullin1-F-box) type of E3 ubiquitin ligase complexes. In the present study, we investigated the role of Skp2 in paclitaxel-resistant DU145-TxR or PC-3-TxR cells by Skp2 silencing or using Skp2 inhibitors. We first confirmed that Skp2 expression is upregulated in DU145-TxR or PC-3-TxR cells compared with their parental cells DU145 or PC-3, respectively. Knockdown of Skp2 or Skp2 inhibitor treatment in DU145-TxR or PC-3-TxR cells restored paclitaxel sensitivity. E-cadherin was decreased while Vimentin was increased in PC-3-TxR or DU145-TxR cells. In addition, p27 expression was inversely correlated with Skp2 expression in DU145-TxR or PC-3-TxR cells. Moreover, p27 was found to increase in both Skp2 silencing PC-3-TxR and DU145-TxR cells. These results suggest that $\mathrm{Skp} 2$ is associated with prostate cancer cell resistance to paclitaxel. Skp2 may be a potential therapeutic target for drug-resistant prostate cancer.
\end{abstract}

Correspondence to: Dr Jiejun Fu or Professor Jian Zhang, Center for Translational Medicine, Guangxi Medical University, 22 Shuangyong Road, 1416 Pharmacology and Biomedical Sciences Building, Nanning, Guangxi 530021, P.R. China

E-mail: fujiejun@126.com

E-mail: jianzhang008@hotmail.com

Abbreviations: PCa, prostate cancer; Skp2, S-phase kinase associated protein 2; SCF, Skp1-Cullin1-F-box; TxR, paclitaxel resistant; AR, androgen receptor; SKPin $\mathrm{C} 1$, Skp2 inhibitor $\mathrm{C} 1$; EMT, epithelial-mesenchymal transition; CTEN, C-terminal tensin like protein; MEF, mouse embryonic fibroblast

Keywords: prostate cancer, Skp2, paclitaxel resistance,p27,E-cadherin

\section{Introduction}

Prostate cancer ( $\mathrm{PCa})$ is the most common male malignancy in developed countries (1). Androgen deprivation therapy is considered an effective strategy in the treatment of advanced PCa patients (2). Castration-resistant PCa often initially responds to taxane-based chemotherapy, but eventually acquisition of chemoresistance leads to failure of the treatment $(3,4)$. Therefore, it is important to find novel therapeutic targets to improve the treatment of patients with hormone-refractory, drug-resistant prostate cancer.

S-phase kinase associated protein 2 (Skp2), a well-characterized F-box protein, is a crucial component of the SCF (Skp1-Cullin1-F-box) type of E3 ubiqutin ligase complexes (5). $\mathrm{SCF}^{\mathrm{Skp} 2}$ is an E3 ubiquitin ligase for androgen receptor (AR), p27, p21, p57, p130, FoxO1, E-cadherin and other substrate ubiquitination and degradation (6). Importantly, Skp2 is found to be upregulated in various types of cancers including prostate cancer (7). It has been shown that Skp2 protein expression was correlated with tumor stage, histological grade, and recurrence in prostate cancer (7). In addition, prostate tissue-specific overexpression of Skp2 causes hyperplastic and dysplastic changes and low-grade carcinomas in the mouse prostate gland (8). Skp2 knockout mice suppresses PCa development induced by loss of either p19ARF or PTEN (9). Skp2 deficiency restricts $\mathrm{PCa}$ development by triggering Arf-p53-independent cellular senescence through upregulation of p21, p27 and ATF4 in vivo (9). Numerous studies indicate that Skp2 interacts with AR, p27, PTEN, PI3K/Akt and BRCA2 signaling, thus plays critical roles in prostate tumorigenesis (6).

Emerging evidence suggests that Skp2 is also involved in the development of drug resistance in human cancers (10). It has been demonstrated that overexpression of Skp2 is associated with resistance to preoperative doxorubicin-based chemotherapy in primary breast cancer (11). Moreover, Skp2 silencing sensitizes Her2-positive tumors to Herceptin treatment (12). Recently, it has been reported that Skp2 is associated with acquisition of epithelial-mesenchymal transition (EMT) and paclitaxel resistance in breast cancer cells (10). However, the role of Skp2 in paclitaxel resistance of PCa remains unclear. 
In this study, we demonstrated that Skp2 is upregulated in paclitaxel-resistant PCa cells DU145-TxR or PC-3-TxR. We found that knockdown of Skp2 restored paclitaxel sensitive in DU145-TxR or PC-3-TxR cells. Importantly, p27, a cyclin dependent kinase inhibitor, was found to be decreased in DU145-TxR or PC-3-TxR cells while increased in Skp2 silencing DU145-TxR or PC-3-TxR cells. We provided evidence that Skp2 is a promising therapeutic target for treatment of hormone-refractory, drug-resistant prostate cancer.

\section{Materials and methods}

Cell culture andreagents. PC3 andDU145 human PCa cells were purchased from ATCC and cultured in RPMI-1640 medium supplemented with $10 \%$ fetal bovine serum and penicillin/ streptomycin (Invitrogen, Carlsbad, CA, USA). Paclitaxelresistant PC-3-TxR and DU145-TxR cells were generated as previously described (3). PC-3-TxR and DU145-TxR cells were cultured in RPMI-1640 medium with $10 \mathrm{nM}$ paclitaxel (Abcam, ab120143) to maintain their drug-resistant phenotypes. Before each experiment, these cells were grown for at least one day in normal medium. HEK293 human embryonic kidney cells were obtained from ATCC and cultured in Dulbecco's modified Eagle's medium supplemented with $10 \%$ fetal bovine serum. Skp2 inhibitor C1 (SKPin C1) was purchased from MedChem Express and SZL-P1-41 (compound \#25) from TOCRIS.

shRNA-mediated silencing. For lentiviral shRNA infection, 293T cells were transfected with Skp2 or control shRNA along with packing plasmids and envelope plasmid using Lipofectamine 2000 reagents according to the manufacturer's instructions. When $293 \mathrm{~T}$ cells were cultured to $80-90 \%$ confluence in a $10-\mathrm{cm}$ dish, the shuttle plasmid of insertion sequences and packaging plasmids (pGag/Pol, pRev, pVSV-G) were transfected and cultured for $72 \mathrm{~h}$, then virus was collected. Skp2 shRNA sequence and negative control sequence was 5'-GGGAGTGACAAAGACTTTG-'3 and 5'-TTCTCCGAACGTGTCACGT-'3, respectively. Real-time PCR and western blotting were used to confirm the efficiency of Skp2 interference.

Cell viability assay. Cells were seeded at a density of $3 \times 10^{3}$ cells/well in 96-well plates containing $200 \mu \mathrm{l}$ of complete medium in five replicate wells. Cells were allowed to attach overnight before treating with the indicated dose of paclitaxel for $24,48,72$ or $96 \mathrm{~h}$. Subsequently, viable cells were treated with $0.3 \mathrm{mg} / \mathrm{ml}$ of MTS for $2 \mathrm{~h}$ and MTS conversion was analyzed by Enzyme-Labeled Instrument (Thermo Fisher Multiskan, GO, USA) at $490 \mathrm{~nm}$.

Real-time quantitative RT-PC. Total RNAs were extracted from cells using TRIzol reagent (Invitrogen) according to the manufacturer's instructions and treated with RNase-free DNase. Reverse transcription reaction was performed on $1 \mu \mathrm{g}$ of total RNA per sample using the SuperScript III reverse transcriptase kit (Invitrogen) according to the manufacturer's protocol. After reverse transcription, the real-time polymerase chain reaction (PCR) was performed using the Power SYBR Green PCR MasterMix (Applied Biosystems, Foster City, CA, USA) on the ABI 7500 thermocycler (Applied Biosystems) following the instrument manual. The sequences of the primers are as follows: GAPDH (sense: 5'-TTCATTGACCTCAACTA CAT-3', antisense: 5'-GAGGGGCCATCCACAGTCTT-3'. Skp2 (sense: 5'-GCTGCTAAAGGTCTCTGGTGT-3', antisense: 5'-AGGCTTAGATTCTGCAACTTG-3'). E-cadherin (sense: 5'-ACGCATTGCCACATACA-3', antisense: 5'-CGT TAGCCTCGTTCTCA-3') Vimentin (sense: 5'-ATGGCTCG TCACCTTCG-3', antisense: 5'-AGTTTCGTTGATAAC CTGTCC-3').

Western blot analysis. Cell lysates were prepared with RIPA buffer (PBS, 1\% Nonidet P40, 0.5\% sodium deoxycholate, $0.1 \%$ SDS and protease inhibitor cocktail (Roche). The proteins were separated by SDS-PAGE and then electrotransferred to membranes. Membranes were blocked in 5\% non-fat milk in TBST (10 mM Tris- $\mathrm{HCl} \mathrm{pH} 8.0,150 \mathrm{mM} \mathrm{NaCl}$, $0.1 \%$ Tween-20) for $1 \mathrm{~h}$ at room temperature. Membranes were then incubated with primary antibody overnight at $4^{\circ} \mathrm{C}$. After incubation with a secondary horseradish peroxidase (HRP)-linked antibody, immunoreactive proteins were detected by using enhanced chemiluminescence supersignal substrate (Thermo/Pierce, Rockford, IL, USA). The following antibodies were used for western blot analysis: p21 (Cell Signaling, 2947), p27 (Cell Signaling, 2552), E-cadherin (Cell Signaling, 3195), Vimentin (Cell Signaling, 5741), FoxO1 (Cell Signaling, 2880), FoxO4 (Cell Signaling, 9472), CyclinB1 (Cell Signaling, 4138), CyclinD1 (Cell Signaling 2926), CyclinE2 (Cell Signaling, 4132), $\beta$-catenin (Cell Signaling, 9562), Skp2 (Invitrogen-187334), $\beta$-actin (Sigma, A1978), Fbxw8 (Sigma,HPA038851), Cul1 (Sigma, C7117), Cul2 (Sigma, SAB4200207), Cul7 (Sigma, C1743), Cul4A (Sigma, C037), Cul4B (Sigma, C9995).

Statistical analysis. Results are presented as means \pm SD. Statistical analyses were performed using the Statistical Package for Social Science (SPSS for Windows package release 10.0; SPSS Inc., Chicago, IL, USA). P $<0.05$ was considered as statistically significant.

\section{Results}

Assessment of paclitaxel sensitivity in paclitaxel-resistant PCa cells. To investigate the molecular mechanisms underlying paclitaxel resistance of hormone-refractory prostate cancer, we first reconfirmed the paclitaxel resistance of DU145-TxR or PC-3-TxR cells. PC3, PC3-TxR, DU145 and DU145-TxR cells were treated with different concentration of paclitaxel for 24, 48, 72 and $96 \mathrm{~h}$ and cell viability was determined by MTT assay. As shown in Fig. 1, as related to vehicle-treated cells, the paclitaxel treatment was statistically significant at $12.5 \mathrm{nM}$ for the 48 and $72 \mathrm{~h}$ treatment in PC-3 cells (Fig. 1A) and that at $200 \mathrm{nM}$ of paclitaxel for the 48 and $72 \mathrm{~h}$ treatment in PC-3-TxR cells (Fig. 1B). The paclitaxel treatment was effective at the dose of $12.5 \mathrm{nM}$ for 48 and $72 \mathrm{~h}$ treatment in DU145 (Fig. 1C) and that at $1600 \mathrm{nM}$ of paclitaxel for the 48 and $72 \mathrm{~h}$ treatment in DU145-TxR cells (Fig. 1D). The $72 \mathrm{~h}$ of $\mathrm{IC}_{50}$ values of parental cells PC-3 or DU145 were 18.42 and $10.91 \mathrm{nM}$, respectively. Cell growth inhibition assay demonstrated that these PC-3-TxR and DU145-TxR cells become 9.04-fold ( $\left.\mathrm{IC}_{50}: 166.47 \mathrm{nM}\right)$ and 
A

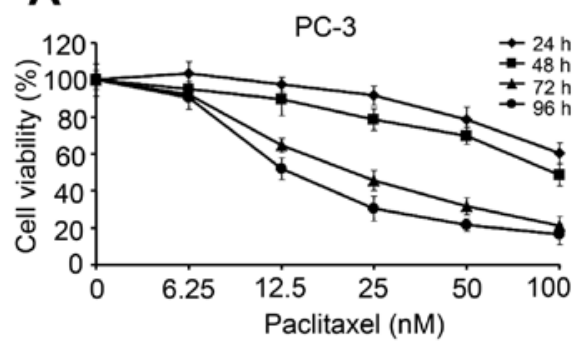

C

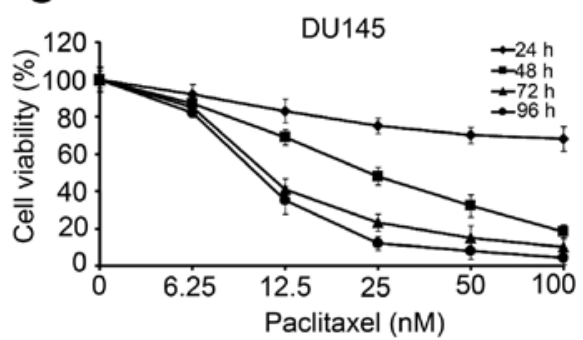

B

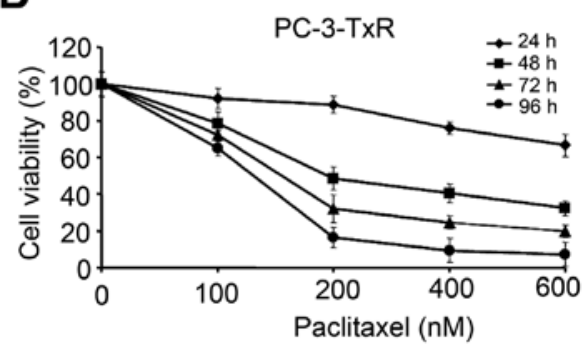

D

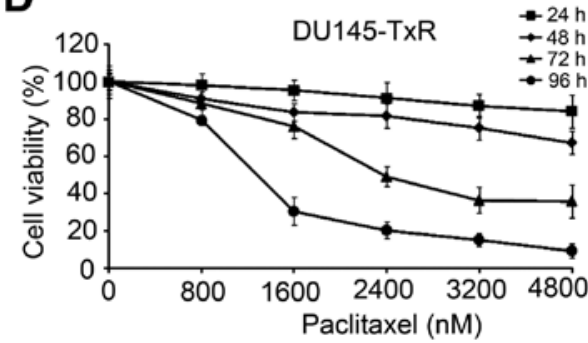

Figure 1. Effects of paclitaxel on cell viability in paclitaxel-resistant PCa cells and their parental cells. Cell viability of PC-3 cells (A) and PC-3-TxR cells (B) treated with increasing doses of paclitaxel for 24, 48, 72 and $96 \mathrm{~h}$ measured by MTT assay. Cell viability of DU145 cells (C) and DU145-TxR cells (D) treated with increasing doses of paclitaxel for 24, 48, 72 and $96 \mathrm{~h}$ measured by MTT assay. Data are presented as mean \pm SD relative to vehicle control of three independent experiments.
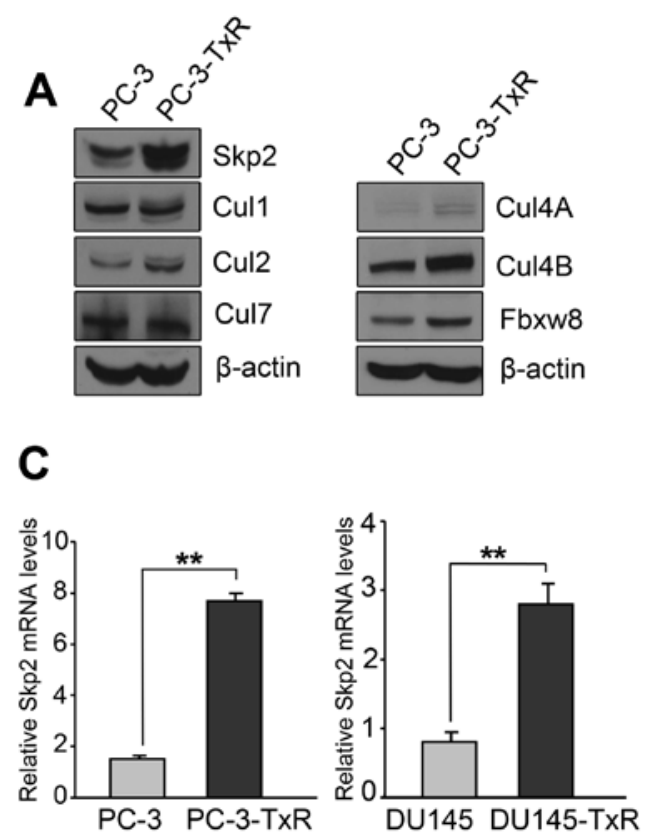

B
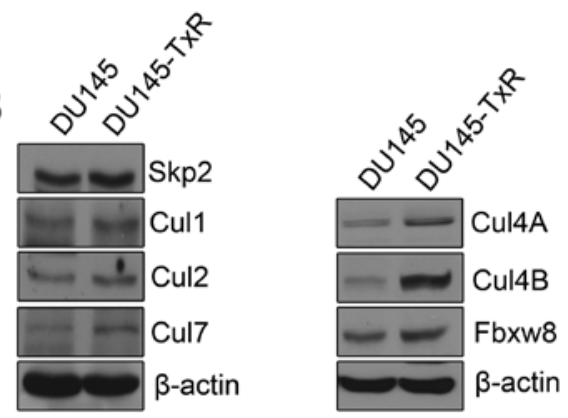

D

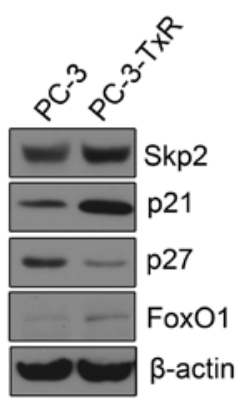

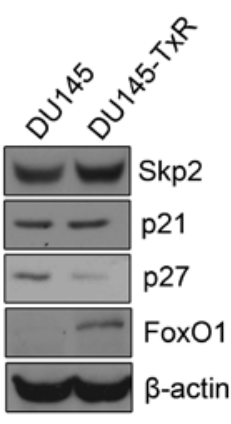

Figure 2. Skp2 is upregulated in paclitaxel resistant PCa cells. (A) Western blotting was performed to detect the expression of Skp2, Cul1, Cul2, Cul7, Cul4A, Cul4B, Fbxw8 in PC-3 and PC-3-TxR cells. (B) Western blotting was performed to detect the expression of Skp2, Cul1, Cul2, Cul7, Cul4A, Cul4B, Fbxw8 in DU145 and DU145-TxR cells. (C) Real-time PCR assay was conducted to detect the expression of Skp2 in parental and paclitaxel resistant PCa cells. (D) Western blotting was performed to detect the expression of Skp2, p21, p27 and FoxO1 in PC-3, PC-3-TxR, DU145 and DU145-TxR cells. $\beta$-actin protein served as loading controls. Bar graphs represent mean \pm SD of three independent experiments. $\left.{ }^{* *} \mathrm{P}<0.01\right)$.

297.49-fold ( $\mathrm{IC}_{50}: 3245.63 \mathrm{nM}$ ) higher than that in both parent cells, respectively.

Skp2 was significantly elevated in paclitaxel-resistant PCa cells. Emerging evidence has demonstrated that Skp2 is involved in drug resistance in human cancer $(10,11)$. To explore whether Skp2 has a critical role in paclitaxel-resistant PCa cells, we measured the expression of Skp2 and other Cullin-
RING E3 ligase components in paclitaxel-resistant cells and parental cells. We found that the expression of Skp2 was significantly elevated in paclitaxel-resistant cells compared with parental cells (Fig. 2A and B). Cul4A, Cul4B, Fbxw8 were also significantly elevated in paclitaxel-resistant cells compared with parental cells. We found that Skp2 at mRNA level was significantly elevated in paclitaxel-resistant cells compared with parental cells (Fig. 2C). On the contrary, 
A

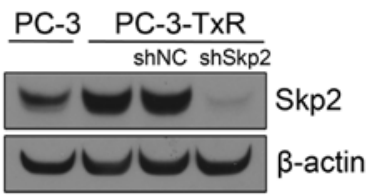

B

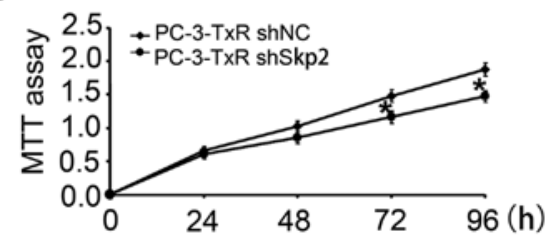

C

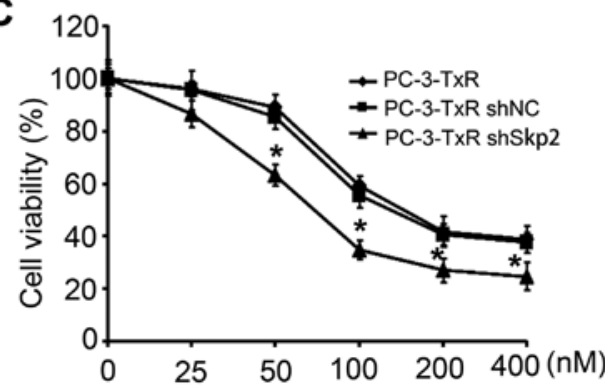

DU145 DU145-TxR
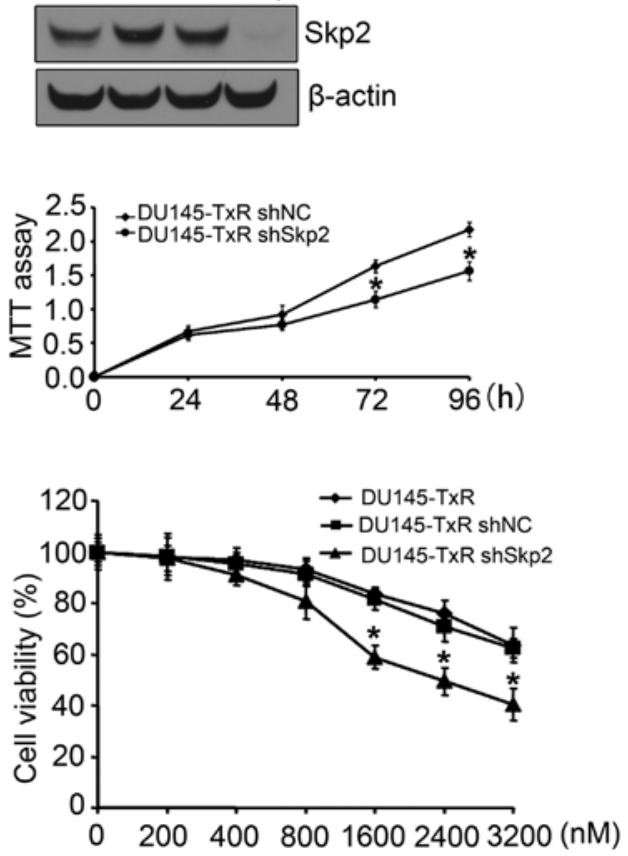

Figure 3. Knockdown of Skp2 increases sensitivity to paclitaxel in paclitaxel-resistant PCa cells. (A) Western blotting was performed to detect the expression of Skp2. (B) MTT assay was performed to detect cell growth inhibition of Skp2 silencing PC3-TxR and DU145-TxR cells. (C) Cell viability of Skp2 silencing PC3-TxR and DU145-TxR cells treated with increasing doses of paclitaxel for $72 \mathrm{~h}$ were measured by MTT assay. Data are presented as mean \pm SD relative to vehicle control of three independent experiments $\left({ }^{*} \mathrm{P}<0.05 ;{ }^{* *} \mathrm{P}<0.01\right)$.

A
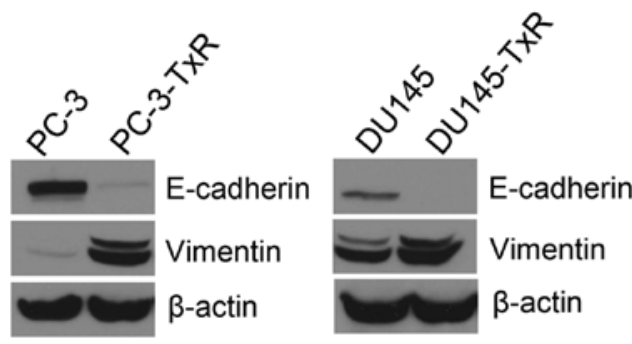

C

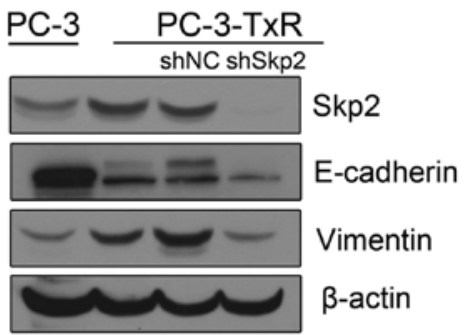

B
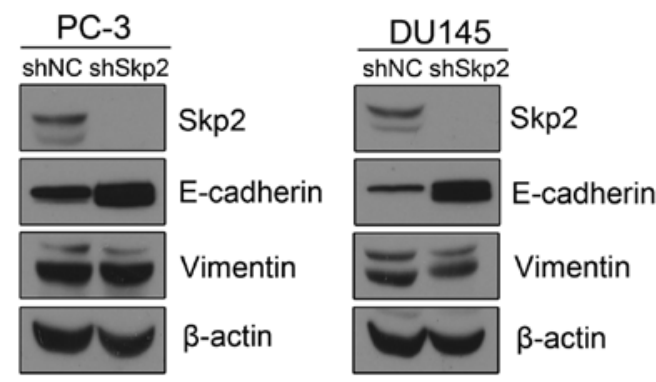

Figure 4. Knockdown of Skp2 does not restore EMT phenotype of paclitaxel resistant PCa cells. (A) Western blotting was performed to detect the expression of E-cadherin and Vimentin in paclitaxel-resistant PCa cells and their parental cells. (B) Western blotting was performed to detect the expression of Skp2, E-cadherin and Vimentin in Skp2 silencing PC-3 and DU145 cells. (C) Western blotting was performed to detect the expression of Skp2, E-cadherin, Vimentin in Skp2 silencing PC-3-TxR and DU145-TxR cells.

p27, one of the Skp2 substrates, was significantly decreased in paclitaxel-resistant cells compared with parental cells (Fig. 2D).

Knockdown of Skp2 enhances sensitivity of paclitaxelresistant cells to paclitaxel. We next investigated whether
Skp2 is involved in the development of paclitaxel resistance in PCa cells. As demonstrated in Fig. 3A, expression of Skp2 was significantly inhibited by Skp2 shRNA transfection in both PC3-TxR and DU145-TxR cells. Thus, we determined the effect of downregulation of Skp2 on paclitaxel sensitivity in PC3-TxR or DU145-TxR cells. Knockdown of Skp2 
A

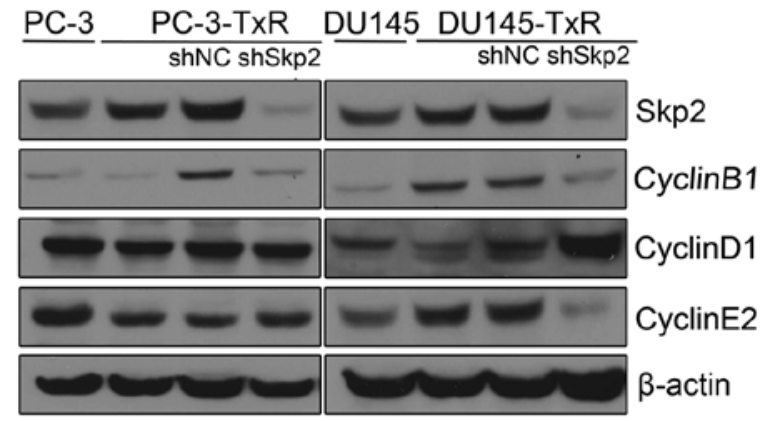

B

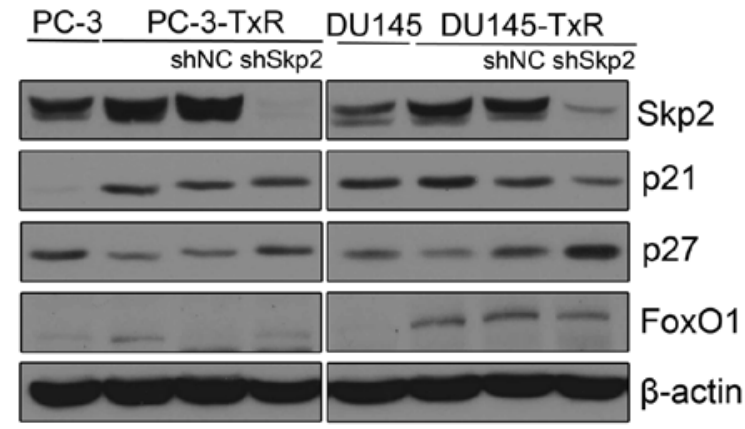

Figure 5. Expression of Skp2-related cell cycle proteins in Skp2 silencing paclitaxel resistant PCa cells. (A) Western blotting was performed to detect the expression of Skp2, CyclinB1, CyclinD1, CyclinE2, p21, p27, FoxO1 in PC-3, PC-3-TxR and Skp2 silencing PC-3-TxR cells. (B) Western blotting was performed to detect the expression of Skp2, CyclinB1, CyclinD1, CyclinE2, p21, p27, FoxO1 in DU145, DU145-TxR and Skp2 silencing DU145-TxR cells.

induced cell growth inhibition of PC3-TxR or DU145-TxR cells (Fig. 3B). Moreover, we found that knockdown of Skp2 significantly enhances paclitaxel-resistant cells to paclitaxel sensitivity (Fig. 3C).

Expression of E-cadherin is not changed in Skp2 silencing paclitaxel-resistant PCa cells. It has been reported that Skp2 is involved in regulation of EMT in paclitaxel-resistant breast cancer cells (10). We found that E-cadherin was significantly decreased whereas the expression of Vimentin was highly elevated in paclitaxel-resistant PCa cells (Fig. 4A). To determined whether knockdown of Skp2 restores E-cadherin expression in paclitaxel-resistant PCa cells, we examined the expression of E-cadherin and Vimentin in Skp2 silencing PC-3, DU145, PC3-TxR and DU145-TxR cells. Knockdown of Skp2 increased E-cadherin expression in PC-3 or DU145 cells (Fig. 4B). However, E-cadherin showed no significant change upon knockdown of Skp2 in PC-3-TxR or DU145-TxR cells (Fig. 4C).

Knockdown of Skp2 restores the expression of p27 in paclitaxel-resistant PCa cells. To investigate the mechanisms by which upregulated Skp2 promotes paclitaxel resistance in $\mathrm{PCa}$ cells, we examined the expression of Skp2-related cell cycle proteins by western blot analysis. We observed that CyclinD1 was increased while CyclinB1 and CyclinE2 decreased in Skp2 silencing DU145-TxR cells, but not in Skp2 silencing PC-3-TxR cells (Fig. 5A). On the contrary, p27, an inhibitor of cyclin-dependent kinases, was found to increase in both Skp2 silencing PC-3-TxR and DU145-TxR cells (Fig. 5B). These data indicated that Skp2-p27 pathway is associated with the development of paclitaxel resistance.

Skp2 inhibitors reverse the resistance of paclitaxel-resistant PCa cells to paclitaxel. Skp2 inhibitor C1(SKPin C1) or SZL-P1-41 (compound \#25) are potent inhibitors of Skp2 and selectively inhibit Skp2-mediated p27 degradation $(32,33)$. To determine the role of Skp2 inhibitors in the development of paclitaxel resistance in $\mathrm{PCa}$ cells, we examined the effects of SKPin C1 and compound \#25 on paclitaxel sensitivity in PC3-TxR or DU145-TxR cells. As shown in Fig. 6, PC3-TxR or DU145-TxR cells were treated with increasing concentrations of SKPin C1 or compound \#25 for 48 h. Both SKPin C1 and compound \#25 suppressed the proliferation of PC3-TxR or DU145-TxR cells in a dose-dependent manner (Fig. 6A). Next, SKPin C1 $(2 \mu \mathrm{M})$ or compound \#25 $(4 \mu \mathrm{M})$ were used to treat PC3-TxR or DU145-TxR cells with increasing concentrations of paclitaxel. We found that both SKPin $\mathrm{C} 1$ and compound \#25 significantly enhances paclitaxel-resistant cells to paclitaxel sensitivity (Fig. 6B).

Furthermore, paclitaxel or Skp2 inhibitor treatment alone led to the accumulation of p27 in PC3-TxR or DU145-TxR cells. Importantly, there is a synergetic effect of the accumulation of p27 in the combination treatment of paclitaxel with Skp2 inhibitors (Fig. 6C). A proposed pathway is shown in (Fig. 6D).

\section{Discussion}

Paclitaxel is usually applied for the treatment of hormonerefractory prostate cancer. However, acquired paclitaxel resistance is a major limitation to improve the treatment of advanced prostate cancer (3). In this study, overexpression of Skp2 was observed in paclitaxel-resistant $\mathrm{PCa}$ cells DU145-TxR or PC-3-TxR. Knockdown of Skp2 in DU145-TxR or PC-3-TxR cells restored paclitaxel sensitivity. Moreover, p27 was found to increase in both Skp2 silencing PC-3-TxR and DU145-TxR cells.

Several important molecules and signaling pathways have been shown to contribute to paclitaxel resistance of PCa cells. The C-terminal tensin like protein (CTEN, tensin 4) gene was downregulated 10-fold in PC-3-TxR cells. Downregulation of CTEN mediates paclitaxel resistance through increasing expression of EGFR and actin (13). Overexpression of Sonic Hedgehog $(\mathrm{SHH})$ increases $\mathrm{PCa}$ cell resistance to paclitaxel and leads to increase in ATP-binding cassette (ABC) transporters expression $(14,15)$. Recently, it has been reported that Sex determining region Y-box 2 (Sox2) is involved in paclitaxel resistance of PC-3-TxR cells via the PI3K/Akt pathway (16). Furthermore, MiR-148a or MiR-34a attenuates paclitaxel resistance of PC-3-TxR cells by regulating MSK1 or SIRT1 expression, respectively $(4,17)$. Emerging evidence also showed that EMT plays an important role in the development of paclitaxel resistance in various cancer including prostate 


\section{A}
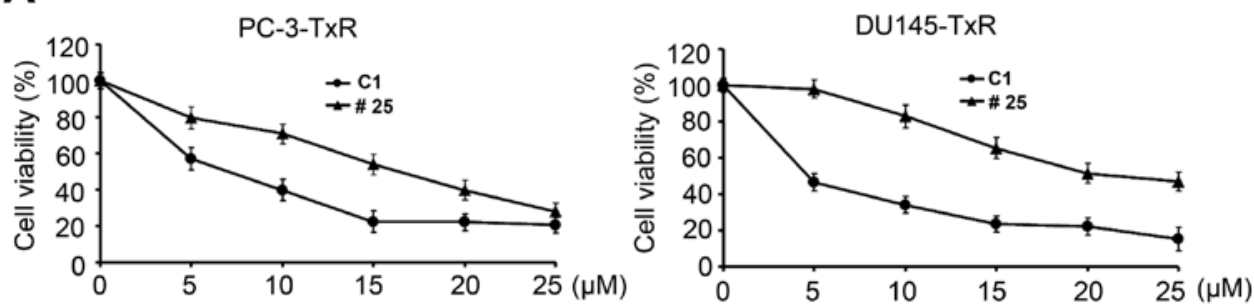

B
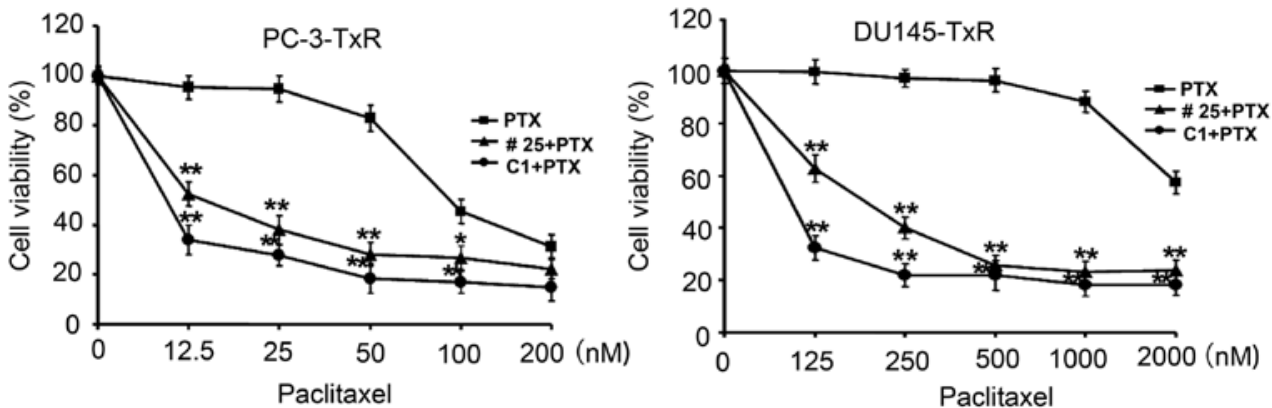

C

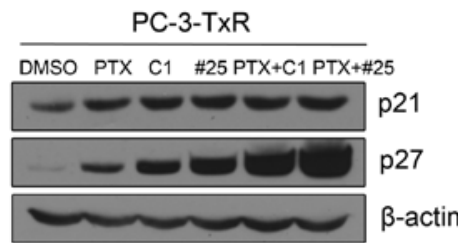

DU145-TxR

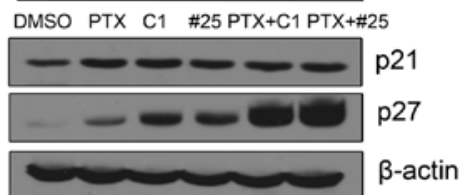

D

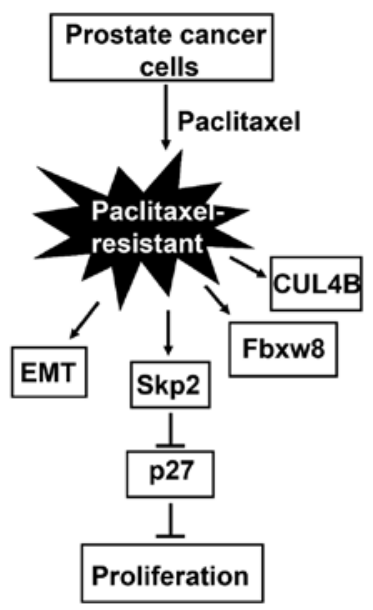

Figure 6. Skp2 inhibitors reverse the resistance of paclitaxel-resistant PCa cells to paclitaxel. (A) Cell viability of PC-3-TxR cells and DU145-TxR cells treated with increasing doses of SKPin C1 or compound \#25 for $72 \mathrm{~h}$ were measured by MTT assay. (B) Cell viability of PC-3-TxR cells and DU145-TxR cells treated with increasing doses of paclitaxel alone or combination treatment for $72 \mathrm{~h}$ were measured by MTT assay. (C) Western blotting was performed to detect the expression of p21, p27 in PC-3-TxR cells and DU145-TxR cells treated with paclitaxel or Skp2 inhibitors alone or their combination treatment. (D) A proposed model for Skp2-p27 pathway in the development of paclitaxel resistance in PCa cells. Data are presented as mean \pm SD relative to vehicle control of three independent experiments ( $\left.\mathrm{P}<0.05 ;{ }^{* * *} \mathrm{P}<0.01\right)$.

cancer $(10,18)$. Nevertheless, the molecular mechanisms responsible for paclitaxel-resistance has not been fully elucidated.

Cullin-RING ligases are the largest family of E3 ubiquitin ligases. There are seven cullin members, Cul1, Cul2, Cul3, Cul4A, Cul4B, Cul5, and Cul7 in human (19). In this study, in addition to Skp2, expression of Cullin-RING ligase components Cul4A, Cul4B, Fbxw8 were also found to be increased in DU145-TxR or PC-3-TxR cells. Activation of Cullin-RING
E3 ligases requires cullin neddylation. MLN4924, a small molecule inhibitor of the NEDD8-activating enzyme (NAE), effectively inhibits cullin neddylation and then inactivates Cullin-RING E3 ligases (20). It has been demonstrated that neddylation inactivation by MLN4924 inhibited Cullin-RING E3 ligases activity of PCa cells, thus induced the accumulation of Cullin-RING E3 ligase ubiquitination substrates, including p21, p27, WEE1, I $\kappa B \alpha$ and DNA replication licensing proteins 
CDT1 and ORC1 (20). Therefore, it will be particularly interesting to determine whether MLN4924 could restore paclitaxel resistance of PCa cells. Furthermore, targeting the Skp2-SCF (Skp1-Cul1-F-box) complex can be an effective strategy for the treatment of cancer. Importantly, Cul1 neddylation is an ideal target to disrupt the Skp2-SCF complex formation (21). MLN4924 also induced senescence in PCa cells by inhibiting Cullin-RING E3 ligases activity and causing p21/p27 accumulation. Moreover, in xenograft tumor models, the growth of PC3 tumors treated with MLN4924 in vivo was also suppressed (21). Therefore, inhibition of neddylation pathway and Cullin-RING E3 ligases could exert potent anticancer efficacy in PCa cells. Noteworthy, MLN4924 is currently in several phase I clinical trials (20). It is critical to explore the role and mechanism of MLN4924 in the development of paclitaxel resistance in $\mathrm{PCa}$ cells.

EMT has been implicated in cancer metastasis and therapeutic resistance in recent years $(10,22)$. Emerging evidence indicates that EMT plays crucial roles during the development of castration-resistance and metastasis of prostate cancer (23). EMT has been shown to be induced by androgen deprivation therapy in both normal prostate and prostate cancer (23). Recently, EMT has also been indicated to be involved in acquiring drug resistance (10). Accumulating evidence suggests that EMT was found to be associated with the resistance of ovarian carcinoma epithelial cells and breast cancer cells to paclitaxel $(10,24)$. More recently, it has been shown that DU145-TxR cells exhibited EMT phenotype and became highly invasive and motile as well as increased tumor growth in mouse xenografts. E-cadherin, Keratin 8, 18, 19 were downregulated while Vimentin, ZEB1 and Snail upregulated in DU145-TxR cells compared with DU145 cells (18). Consistent with this notion, we also found that PC3-TxR and DU145-TxR cells acquired EMT characteristics. It is well accepted that many transcription factors such as Snail, Slug, ZEB1, KLF8 can bind to E-cadherin reporter and repress its expression $(10,23)$.

It has been shown that E-cadherin is destroyed by ubiquitin-proteasome mediated degradation. Of note, E-cadherin is a substrate of Skp2-SCF complex (21). Overexpression of Skp2 has been found to be associated with breast cancer drug resistance and EMT (10). Knockdown of Skp2 in breast cancer cells led to partial reversal of EMT phenotype. We observed that expression of E-cadherin was significantly decreased in paclitaxel-resistant PCa cells DU145-TxR or PC-3-TxR compared with their parental cells DU145 or PC-3, respectively. However, there was no significant change of E-cadherin expression in Skp2 silenced PC-3-TxR or DU145-TxR cells. These results suggest that Skp2 knockdown did not restore the expression of E-cadherin in PC-3-TxR or DU145-TxR cells. Our results suggest that $\mathrm{Skp} 2$ may not be the major regulator for E-cadherin expression in paclitaxel-resistant $\mathrm{PCa}$ cells, thus other factors may contribute to EMT phenotype of PC-3-TxR or DU145-TxR cells. This discrepancy of Skp2 controlled E-cadherin expression between paclitaxel-resistant PCa cells and their parental cells is not clear.

The best known Skp2 ubiquitination substrate is p27, an inhibitor of cyclin-dependent kinases (7). It is well established that Skp2 plays a critical role in controlling the cell cycle through the G1/S transition by promoting the destruction of p27 (25). Accumulated evidence also suggests that Skp2 acts as an oncoprotein in cell proliferation, survival, and cancer development mainly through its degradation of p27 $(6,26)$. Skp2 knockout mouse embryonic fibroblast (MEFs) display reduced cell proliferation, accompanied by enhanced p27 protein expression. Noteworthy, double deficiency for $\mathrm{p} 27$ and Skp2 rescues the cell proliferation defect in Skp2 knockout MEFs and the reduced organ size and body weight observed in Skp2 knockout mice (27,28). Importantly, Skp2 overexpression is found in various human cancer samples associated with poor prognosis and inversely correlated with p27 expression level $(26,29)$.

It has been demonstrated that Skp2 expression was inversely correlated with p27 expression in prostate cancer (29). Moreover, overexpression of Skp2 in transgenic mice significantly decreased p27 protein expression level in prostate glands (8). Of note, in addition to functioning as an inhibitor of cyclin-dependent kinases and a tumor suppressor, altered regulation of $\mathrm{p} 27$ may be involved in resistance to chemotherapy (30). It has been shown that p27 is associated with chemotherapeutic drug resistance in cancer including gastric cancer, lung adenocarcionma and breast cancer $(5,30)$. In this study, we found that expression of p27 was significantly decreased while Skp2 overexpressed in paclitaxel-resistant PCa cells DU145-TxR or PC-3-TxR compared with their parental cells DU145 or PC-3, respectively. Importantly, expression level of p27 was significantly reversed by knockdown of Skp2 in both DU145-TxR and PC-3-TxR cells. We propose that Skp2 promotes PCa proliferation and paclitaxel resistance by targeting p27. Further investigation is necessary for elucidating the molecular mechanism of Skp2-p27 pathway in the development of PCa paclitaxel resistance. Taken together, our results strongly suggested that $\mathrm{p} 27$, rather than E-cadherin, was one of the major substrates of Skp2 in paclitaxel-resistant PCa cells DU145-TxR or PC-3-TxR.

Pharmacological Skp2 inactivation could restrain cancer progression in various cancer models including prostate cancer (32). Given that Skp2 is involved in the development of paclitaxel resistance in PCa cells, it would be interesting to test whether Skp2 inhibitors could be applied for combination treatment of paclitaxel-resistant prostate cancer. Using high-throughput screening or in silico approaches, two small molecule inhibitors SKPin C1 or compound \#25 targeting Skp2 mediated p27 ubiquitination have been developed (32). In this study, we observed that SKPin C1 or compound \#25, like Skp2 knockdown, enhances sensitivity of paclitaxelresistant prostate cacner cells to paclitaxel and impairs p27 degradation. Recently, it has been shown that both Skp2 knockdown and compound \#25 suppresses cancer stem cell populations and self-renewal ability (32). Cancer stem cells are known to develop resistance to chemotherapy and considered as one major cause of treatment failure (33). Targeting Skp2 by SKPin C1 or compound \#25 and combined with paclitaxel could be a novel approach for achieving more effective treatment outcome of PCa patients.

In conclusion, we showed that Skp2 was upregulated in paclitaxel-resistant PCa cells. Skp2 inhibition enhanced paclitaxel-resistant DU145-TxR as well as PC-3-TxR cells to paclitaxel sensitivity and reversed the protein expression level of p27. Our results strongly suggests that inactivation 
of Skp2 could be a promising systemic therapy strategy for restoring sensitivity to paclitaxel. Thus, Skp2 may be a potential molecular target for the treatment of advanced prostate cancers with acquired resistance to paclitaxel.

\section{Acknowledgements}

This study was supported by grants from National Natural Science Foundation of China $(81130046,81201669,81171993$, 81272415,81560483 ) and Natural Science Foundation of Guangxi (2013GXNSFAA019211, 2014GXNSFCA118009, 2013GXNSFEA053004，2012GXNSFCB053004, 2013GXNSFBA019177，1355004-5，201201ZD004, GZPT13-35, 14122008-22, 11-031-05-K2, KY2015YB057, 14-045-12-K2) as well as grants from Guangxi Educational Committee (201106LX087, 201203YB034). The authors would like to thank Drs Yong Wan and Chunlin Zou for helpful discussions and Xin Huang for editing.

\section{References}

1. Torre LA, Bray F, Siegel RL, Ferlay J, Lortet-Tieulent J and Jemal A: Global cancer statistics, 2012. CA Cancer J Clin 65: 87-108, 2015

2. Pernicová Z, Slabáková E, Kharaishvili G, Bouchal J, Král M, Kunická Z, Machala M, Kozubík A and Souček K: Androgen depletion induces senescence in prostate cancer cells through down-regulation of Skp2. Neoplasia 13: 526-536, 2011.

3. Takeda M, Mizokami A, Mamiya K, Li YQ, Zhang J, Keller ET and Namiki M: The establishment of two paclitaxel-resistant prostate cancer cell lines and the mechanisms of paclitaxe resistance with two cell lines. Prostate 67: 955-967, 2007.

4. Fujita Y, Kojima K, Ohhashi R, Hamada N, Nozawa Y, Kitamoto A, Sato A, Kondo S, Kojima T, Deguchi T, et al MiR-148a attenuates paclitaxel resistance of hormone-refractory, drug-resistant prostate cancer PC 3 cells by regulating MSK1 expression. J Biol Chem 285: 19076-19084, 2010.

5. Wei W, Ayad NG, Wan Y, Zhang GJ, Kirschner MW and Kaelin WG Jr: Degradation of the SCF component Skp2 in cellcycle phase G1 by the anaphase-promoting complex. Nature 428 : 194-198, 2004.

6. Wang Z, Gao D, Fukushima H, Inuzuka H, Liu P, Wan L, Sarkar FH and Wei W: Skp2: A novel potential therapeutic target for prostate cancer. Biochim Biophys Acta 1825: 11-17, 2012.

7. Drobnjak M, Melamed J, Taneja S, Melzer K, Wieczorek R, Levinson B, Zeleniuch-Jacquotte A, Polsky D, Ferrara J, Perez-Soler R, et al: Altered expression of p27 and Skp2 proteins in prostate cancer of African-American patients. Clin Cancer Res 9: 2613-2619, 2003.

8. Shim EH, Johnson L, Noh HL, Kim YJ, Sun H, Zeiss C and Zhang H: Expression of the F-box protein SKP2 induces hyperplasia, dysplasia, and low-grade carcinoma in the mouse prostate. Cancer Res 63: 1583-1588, 2003.

9. Lin HK, Chen Z, Wang G, Nardella C, Lee SW, Chan CH, Yang WL, Wang J, Egia A, Nakayama KI, et al: Skp2 targeting suppresses tumorigenesis by Arf-p53-independent cellular senescence. Nature 464: 374-379, 2010.

10. Yang Q, Huang J, Wu Q, Cai Y, Zhu L, Lu X, Chen S, Chen C and Wang Z: Acquisition of epithelial-mesenchymal transition is associated with Skp2 expression in paclitaxel-resistant breast cancer cells. Br J Cancer 110: 1958-1967, 2014.

11. Davidovich S, Ben-Izhak O, Shapira M, Futerman B and Hershko DD: Over-expression of Skp2 is associated with resistance to preoperative doxorubicin-based chemotherapy in primary breast cancer. Breast Cancer Res 10: R63, 2008.

12. Chan CH, Li CF, Yang WL, Gao Y, Lee SW, Feng Z, Huang HY, Tsai KK, Flores LG, Shao Y, et al: The Skp2-SCF E3 ligase regulates Akt ubiquitination, glycolysis, herceptin sensitivity, and tumorigenesis. Cell 149: 1098-1111, 2012.

13. Li Y, Mizokami A, Izumi K, Narimoto K, Shima T, Zhang J, Dai J, Keller ET and Namiki M: CTEN/tensin 4 expression induces sensitivity to paclitaxel in prostate cancer. Prostate 70 $48-60,2010$
14. Statkiewicz M, Maryan N, Lipiec A, Grecka E, Grygorowicz MA, Omiotek M, Gorska A, Mikula M and Malecki M: The role of the SHH gene in prostate cancer cell resistance to paclitaxel. Prostate 74: 1142-1152, 2014

15. Singh S, Chitkara D, Mehrazin R, Behrman SW, Wake RW and Mahato RI: Chemoresistance in prostate cancer cells is regulated by miRNAs and Hedgehog pathway. PLoS One 7: e40021, 2012.

16. Li D, Zhao LN, Zheng XL, Lin P, Lin F, Li Y, Zou HF, Cui RJ, Chen $\mathrm{H}$ and Yu XG: Sox 2 is involved in paclitaxel resistance of the prostate cancer cell line PC-3 via the PI3K/Akt pathway. Mol Med Rep 10: 3169-3176, 2014.

17. Kojima K, Fujita Y, Nozawa Y, Deguchi T and Ito M: MiR-34a attenuates paclitaxel-resistance of hormone-refractory prostate cancer PC3 cells through direct and indirect mechanisms. Prostate 70: 1501-1512, 2010.

18. Kim JJ, Yin B, Christudass CS, Terada N, Rajagopalan K, Fabry B, Lee DY, Shiraishi T, Getzenberg RH, Veltri RW, et al: Acquisition of paclitaxel resistance is associated with a more aggressive and invasive phenotype in prostate cancer. J Cell Biochem 114: 1286-1293, 2013.

19. Sarikas A, Hartmann T and Pan ZQ: The cullin protein family. Genome Biol 12: 220-232, 2011.

20. Wang X, Li L, Liang Y, Li C, Zhao H, Ye D, Sun M, Jeong LS, Feng Y, Fu S, et al: Targeting the neddylation pathway to suppress the growth of prostate cancer cells: Therapeutic implication for the men's cancer. Biomed Res Int 2014: 974309, 2014.

21. Inuzuka H, Gao D, Finley LW, Yang W, Wan L, Fukushima H, Chin YR, Zhai B, Shaik S, Lau AW, et al: Acetylation-dependent regulation of Skp2 function. Cell 150: 179-193, 2012.

22. Li P, Yang R and Gao WQ: Contributions of epithelialmesenchymal transition and cancer stem cells to the development of castration resistance of prostate cancer. Mol Cancer 13: 55, 2014.

23. Sun Y, Wang BE, Leong KG, Yue $P$, Li L, Jhunjhunwala $S$, Chen D, Seo K, Modrusan Z, Gao WQ, et al: Androgen deprivation causes epithelial-mesenchymal transition in the prostate: Implications for androgen-deprivation therapy. Cancer Res 72: 527-536, 2012.

24. Kajiyama H, Shibata K, Terauchi M, Yamashita M, Ino K, Nawa A and Kikkawa F: Chemoresistance to paclitaxel induces epithelial-mesenchymal transition and enhances metastatic potential for epithelial ovarian carcinoma cells. Int J Oncol 31: 277-283, 2007.

25. Zhao H, Bauzon F, Fu H, Lu Z, Cui J, Nakayama K, Nakayama KI, Locker J and Zhu L: Skp2 deletion unmasks a p27 safeguard that blocks tumorigenesis in the absence of $\mathrm{pRb}$ and $\mathrm{p} 53$ tumor suppressors. Cancer Cell 24: 645-659, 2013.

26. Zheng XY, Ding W, Xie LP and Chen ZD: Correlation of Skp2 and P27kip1 protein expression and clinicopathological features of prostate cancer. Ai Zheng 23: 215-218, 2004 (In Chinese).

27. Nakayama K, Nagahama $\mathrm{H}$, Minamishima YA, Matsumoto $M$, Nakamichi I, Kitagawa K, Shirane M, Tsunematsu R, Tsukiyama T, Ishida N, et al: Targeted disruption of Skp2 results in accumulation of cyclin $\mathrm{E}$ and $\mathrm{p} 27$ (Kip1), polyploidy and centrosome overduplication. EMBO J 19: 2069-2081, 2000.

28. Zhu L: Skp2 knockout reduces cell proliferation and mouse body size: And prevents cancer? Cell Res 20: 605-607, 2010.

29. Ben-Izhak O, Lahav-Baratz S, Meretyk S, Ben-Eliezer S, Sabo E, Dirnfeld M, Cohen S and Ciechanover A: Inverse relationship between Skp2 ubiquitin ligase and the cyclin dependent kinase inhibitor p27Kip1 in prostate cancer. J Urol 170: 241-245, 2003.

30. Le TV, Seo Y, Ryu CJ, Lee HR and Park HJ: Increased expression of p27 is associated with the cisplatin resistance in gastric cancer cell line YCC-3. Arch Pharm Res 33: 1127-1132, 2010.

31. Zhao YF, Zhao JY, Yue H, Hu KS, Shen H, Guo ZG and Su XJ: FOXD1 promotes breast cancer proliferation and chemotherapeutic drug resistance by targeting p27. Biochem Biophys Res Commun 456: 232-237, 2015.

32. Chan CH, Morrow JK, Li CF, Gao Y, Jin G, Moten A, Stagg LJ, Ladbury JE, Cai Z, Xu D, et al: Pharmacological inactivation of Skp2 SCF ubiquitin ligase restricts cancer stem cell traits and cancer progression. Cell 154: 556-568, 2013.

33. Chan CH, Morrow JK, Zhang S and Lin HK: Skp2: A dream target in the coming age of cancer therapy. Cell Cycle 13: 679-680, 2014. 